\title{
Daño por suplementos alimenticios y herbales: un problema emergente para la salud pública
}

\section{Harm from food and herbal supplements: An emerging problem for public health}

\author{
Iván R. Zúñiga-Carrasco*
}

Departamento de Epidemiología, Unidad de Medicina Familiar 223, Instituto Mexicano del Seguro Social, Lerma de Villada, Estado de México, México

\begin{abstract}
Resumen
Antecedentes: Los suplementos herbales y dietéticos se usan comúnmente en todo el mundo, ya sea suplantando la terapéutica médica convencional o a manera de complementar el tratamiento. Sin embargo, se han observado casos de daño hepático por el consumo de dichos suplementos. Objetivos: Realizar una revisión bibliográfica sobre los daños que provocan los suplementos alimenticios y herbales, generando un problema emergente para la salud pública. Material y métodos: Se realizó una búsqueda selectiva de literatura en PubMed, Medscape y Google Académico utilizando términos tales como: suplementos y lesión hepática, lesión hepática inducida por hierbas, hepatotoxicidad relacionada con el consumo de productos de Herbalife ${ }^{\circ}$. La búsqueda fue enfocada principalmente en informes de casos, series de casos y revisiones clínicas, publicados desde 1984 hasta 2019. Resultados: La mayoría de los reportes están redactados en idioma inglés, en español solo uno. Conclusiones: Existe un severo subregistro a nivel mundial, a pesar de que existen casos reportados por hepatotoxicidad provocados por suplementos dietéticos, lo que apunta a un importante problema de salud pública.
\end{abstract}

Palabras clave: Suplementos dietéticos. Medicina de hierbas. Hepatitis.

\section{Abstract}

Background: Herbal and dietary supplements are commonly used around the world, either supplanting conventional medical therapy or as a supplement to treatment. However, worldwide cases of liver damage have been observed from the consumption of these supplements. Objectives: Carry out a bibliographic review on the damages caused by nutritional and herbal supplements, generating an emerging problem for public health. Material and methods: A selective literature search was conducted in PubMed, MedScape, Google Scholar using terms such as: supplements and liver injury, herbal-induced liver injury, hepatotoxicity related to Herbalife use. The search was mainly focused on case reports, case series and clinical reviews, published from 1984 to 2019. Results: Most of the reports are written in English, only one in Spanish. Conclusions: There is a severe underreporting worldwide, despite the fact that there are reported cases of hepatoxicity caused by dietary supplements, thus pointing to an important public health problem.

Key words: Dietary supplements. Herbal medicine. Hepatitis.

Correspondencia:

*Iván R. Zúñiga-Carrasco

E-mail: ivan.zuniga@imss.gob.mx
Disponible en internet: 13-09-2021 Rev Hosp Jua Mex. 2021;88(3):151-158

www.revistahospitaljuarez.com 1405-9622/@ 2021 Sociedad Médico-Quirúrgica del Hospital Juárez de México, A.C. Publicado por Permanyer. Este es un artículo open access bajo la licencia CC BY-NC-ND (http://creativecommons.org/licenses/by-nc-nd/4.0/). 


\section{Introducción}

En la mayoría de los países de América Latina, sus habitantes, por el número tan alto de enfermedades crónicas y sobre todo por la lucha contra la obesidad, recurren, ya no a remedios caseros hechos empíricamente a base de plantas y hasta levaduras como se hacía hasta hace tres décadas, sino a «efectivos suplementos alimenticios». Estos productos dicen ser de origen $« 100 \%$ natural» y ayudar en dichas enfermedades, inclusive para bajar de peso sin necesidad de cambiar estilos de vida, tan es así que dichos productos los pueden consumir niños y adolescentes «con total seguridad". Los suplementos herbales y dietéticosse usan comúnmente en el mundo, ya sea suplantando la terapéutica médica convencional o a manera de complementar el tratamiento'. A pesar del progreso de la medicina convencional, en los últimos 20 años ha aumentado la popularidad de las hierbas y suplementos dietéticos (SD). Diversos factores contribuyen a la atractiva terapéutica de la medicina a base de hierbas. Dentro de las falacias para el consumo y supuesto éxito "de mejoría» se encuentran: «influyen» sobre la historia natural de una enfermedad determinada, «generan bienestar» físico y emocional, «reducen» los efectos secundarios de medicamentos alopáticos, «mejoran» los síntomas de enfermedades existentes, «fortalecen» al sistema inmunitario, «mejoran» la calidad de vida, tienen «buena» relación terapéutica, el «proceso de curación» es natural y hay «disponibilidad» de tratamiento ${ }^{2}$. Lo antes comentado refleja una creencia profunda, que los suplementos dietéticos y herbales (SDH) son seguros porque son «naturales» $y$ se ajustan a la imagen de una alternativa amable, y por lo tanto inofensiva, a la medicina convencional. Los pacientes están a menudo insatisfechos con el tratamiento alopático convencional, debido a los habituales efectos adversos. Un aspecto positivo es que reflejan una mayor conciencia hacia la salud y el bienestar en general, pero las expectativas hacia las supuestas virtudes de los SDH son tan numerosas como los diversos grupos de usuarios. Los SDH suelen estar excluidos de las estrictas regulaciones de prescripción, por lo tanto se pueden comprar fácilmente en farmacias, tiendas de salud, gimnasios, internet o a un distribuidor de dichos suplementos, en ocasiones siendo relativamente bajo su costo ${ }^{3,4}$. La bibliografía especializada utiliza la frase "suplementos dietéticos y herbales»" para referirse a cualquier suplemento que pueda estar implicado en causar daño hepático, estos productos incluyen suplementos herbales o botánicos, productos como vitaminas, minerales, aminoácidos y proteínas que se utilizan para complementar la dieta, así como suplementos para mejorar el rendimiento, los cuales pueden conteneresteroides anabólicos sintetizados químicamente. Los SDH se han llegado a utilizar para mantener o mejorar la salud; la regulación de productos a base de hierbas puede variar entre los diferentes países. Stickel, et al. han clasificado los SDH relacionados con hepatotoxicidad en dos diferentes grupos: daño hepático inducido por hierbas y daño hepático inducido por SD ${ }^{5}$. Stickel ${ }^{4}$ considera a todo SD si se consume como ayuda para mejorar el estado nutricional, perder peso o tratar el estreñimiento. La Ley de Educación y Salud de Suplementos Dietéticos (Dietary Supplement Health and Education Act) define SD como cualquier producto destinado a complementar, pero no sustituir, la dieta; los SD puede contener uno o más ingredientes, incluidas vitaminas, minerales, hierbas, productos botánicos, aminoácidos o extractos herbales ${ }^{5}$.

\section{Objetivos}

Realizar una revisión bibliográfica sobre los daños que provocan los suplementos alimenticios y herbales generando un problema emergente para la salud pública.

\section{Metodología}

Se realizó una búsqueda selectiva de literatura en PubMed, Medscape y Google Académico utilizando términos tales como: suplementosylesión hepática, lesión hepática inducida por hierbas, hepatotoxicidad relacionada con el consumo de Herbalife ${ }^{\circledR}$. Labúsqueda fue enfocada principalmente en informes de casos, series de casos y revisiones clínicas, publicados desde 1984 hasta 2019.

\section{Criterios}

- Inclusión: artículos relacionados sobre lesión hepática inducida por hierbas, hepatotoxicidad relacionada con el consumo de Herbalife ${ }^{\circledR}$.

- Exclusión: aquellos artículos que no tengan sustento metodológico y que no los avale una institución.

\section{Suplementos dietéticos y lesión hepática}

Un SD no está exento de aspectos necesarios tanto para su producción y venta, como porque es un producto para consumo humano: definición del producto, criterios de eficacia y seguridad, los cuales por lo 
regular están escasamente documentados. Los SD necesitan una definición clara de sus ingredientes, así como cuáles con son los riesgos y beneficios. Así mismo, estos productos no complementan cualquier dieta del individuo que consume regularmente; cuando hay deficiencias dietéticas clínicamente detectadas, existen terapias de sustitución específicas aprobadas y estandarizadas, las cuales están bajo la guía y vigilancia de personal de salud. En este apartado deben descartarse absolutamente consejos de youtubers, influencers, couches, entrenadores de gimnasio, naturistas, proveedores $\mathrm{y} / \mathrm{o}$ vendedores de las marcas Herbalife ${ }^{\circledR}$, Omnilife ${ }^{\circledR}$ y Kromasol $^{\circledR}$ (HOK). Cabe enfatizar que los pacientes con obesidad se enfrentan a comorbilidades, por lo tanto, el consumo habitual de SD no puede considerarse como "saludable» como se ha llegado a suponer. Añadir algún SD al uso concomitante de múltiples medicamentos por los pacientes obesos puede conducir a daño hepático. Algunos medicamentos pueden generar cierta toxicidad farmacológica debido al polimorfismo genético de enzimas xenobióticas, hay que destacar que el genotipo del antígeno leucocitario humano es un fuerte factor de riesgo para el desarrollo de fármacos que inducen daño hepático. Hay que destacar que en los informes de casos de sospecha de daño hepático debido a SD utilizado para la reducción de peso, los datos sobre el índice de masa corporal se encontraron con valores normal o subnormal, ante la suspensión de SD no existe reducción alguna de este por su consumo ${ }^{6}$.

\section{Suplementos dietéticos y herbales comúnmente asociados con lesión hepática}

Es importante destacar que los SDH rara vez contienen un solo ingrediente ${ }^{6}$. La mayoría de los agentes ${ }^{1}$ implicados son mezclas complejas vendidas bajo nombres comerciales. Entre los casos de lesión hepática, se atribuyeron a uno o varios productos herbales, mezclas botánicas tradicionales, vitaminas o minerales, niacina y levocarnitina entre otros componentes. La lesión hepática inducida por SDH abarca un espectro de presentaciones. Un contribuyente potencial de daño hepático importante ${ }^{1}$ es la contaminación del producto, no solo con elementos que pueden ser tóxicos al momento de la manufactura, sino también con otras hierbas desconocidas o la adición de compuestos tales como inhibidores de la 5-fosfodiesterasa (sildenafilo), agentes antiinflamatorios no esteroideos, estatinas y corticosteroides. Destacan otros productos herbales implicados: valeriana (Valeriana officinalis), Eurycoma longifolia, ajenjo, ontina o incienso de Canarias (Artemisia herba-alba), uña de gato (Uncaria tomentosa), Ganoderma applanatum, fo-ti (Fallopia multiflora), arroz de levadura roja (Monascus purpureus), Garcinia cambogia, kratom (Mitragyna speciosa) y el cohosh negro (Cimicifuga racemosa).

\section{Frecuencia de lesión hepática por suplementos dietéticos y herbales: EE.UU. $y$ el mundo}

Aunque no existen estimaciones basadas en la población para la frecuencia de lesiones hepáticas por SDH en los EE.UU., la incidencia tiende al incremento. En un estudio prospectivo con datos obtenidos de la Red de Lesiones Hepáticas Inducidas por Fármacos el consumo de SDH representó el 16\% de los casos en general ${ }^{6}$. Sin embargo, es importante destacar que la proporción aumentó durante los ocho años del estudio del 7\% en 2004-2005 al 19\% en 2010-2012. Desde entonces, la proporción de casos de lesión hepática atribuible a SDH se ha mantenido por arriba del $20 \%$. Los estudios en Europa ${ }^{7,8}$ muestran aumentos en el consumo de SDH en los últimos 10 años. En el registro español de lesiones hepáticas inducidas por fármacos, la proporción de casos atribuidos a SDH fue del $2 \%$ en 2006 y aumentó al 13\% para el periodo 2010-2013, lo anterior representa una tendencia durante un periodo de 10 años de incremento de lesiones hepáticas por el consumo de SDH. Laproporción de casos de lesiones hepáticas atribuidasaSDHvaría en los países asiáticos: el $70 \%$ en Singapur, el $73 \%$ en Corea del Sur, el $18.6 \%$ en China y solo el $2.5 \%$ en India, a pesar del uso de medicinas alternativas o tradicionales en los cuatro países ${ }^{9-12}$. La mejor estimación de la incidencia de daño hepático relacionado con SDH proviene de una encuesta basada en la población en Islandia, donde se estimó que en 20112012 el 16\% de los casos se atribuyeron a SDH, lo que sugiere que la incidencia de lesión hepática aguda relacionada con SDH fue de 3 por cada 100,000 habitantes-estudio $0^{1,13}$.

\section{Hepatotoxicidad relacionada con suplementos dietéticos y herbales}

Los productos herbales y SD de tipo «natural» son populares, en más del $60 \%$ de los personas, los usuarios al percibirlos como "naturales" los consideran seguros. Sin embargo, en la literatura ${ }^{14}$ hay informes de hepatotoxicidad como se comentó anteriormente. El 
tema de la hepatotoxicidad relacionada con los SD es una preocupación creciente, particularmente con la evidencia tanto en EE.UU. como en Europa, principalmente por el consumo de estos productos ${ }^{1}$. Marignani, et al. ${ }^{6}$ investigaron la proporción de pacientes con trastornos hepatobiliares que toman SDH, encontrando que más de un tercio de los sujetos admitieron el uso de SDH, a menudo complementario a la terapéutica convencional ${ }^{15}$; en seis clínicas de EE.UU. incluso se encontraron cifras altas de consulta por enfermedad hepática, revelando que el $65 \%$ de los pacientes con enfermedad hepática consumieron $\mathrm{SDH}^{16}$. Un informe español ${ }^{17}$ reportó 13 casos relacionados con SDH. El uso generalizado de SDH está en gran medida mal regulado, el desconocimiento de sus consumidores y proveedores sobre daños potenciales está provocando importantes subregistros ${ }^{4}$. En EE.UU. existen alrededor de 45,000 casos por $\mathrm{SDH}^{3,4,5,18}$. Esto último es importante, en la práctica clínica diaria del médico general, familiar, así como en la del médico especialista, los pacientes que usan hierbas o SD llegan a experimentar lesión hepática o tienen pruebas de funcionamiento hepático anormales; es importante analizar si el paciente cursa con una enfermedad hepática crónica o es debida por SDH.

Al realizar la historia clínica, los pacientes no comentan el consumo de productos Herbalife ${ }^{\circledR} 0$ de otras marcas, los médicos a menudo no piensan en SDH o no preguntan sobre su consumo, en caso de que lo cuestionen y resulte positivo creen que no pueden provocar daños por "ser naturales». Generalmente los pacientes omiten el uso de tales productos, ya que no los consideran potencialmente dañinos, 0 porque temen no ser tomados en serio por el médico ${ }^{4}$. Realizar un estudio de calidad de los productos de la marca líder en cuestión es extremadamente difícil, por la reticencia de la empresa para revelar la composición de sus diferentes productos. Asimismo, la empresa prepara sus productos de manera diferente en cada país. Histológicamente, de cinco de cada siete pacientes en los que estaba disponible la biopsia: uno manifestó hepatitis colestásica con eosinófilos prominentes e infiltración, otro presentó hepatitis con células gigantes, necrosis subaguda masiva, y el resto de los pacientes manifestó síndrome sinusoidal de obstrucción. Algunos pacientes desarrollaron fibrosis leve a moderada; esto último fue severo en un paciente con un año de ingesta del producto. El pronóstico puede ser favorable si se identifica al agente causal tempranamente y se suspende su consumo. El hígado puede evolucionar a la insuficiencia, poniendo en peligro la vida de la persona; esto puede ocurrir por la ingesta a largo plazo, en particular cuando el paciente no reconoce qué productos está consumiendo.

\section{Suplementos dietéticos y productos herbarios asociados a hepatotoxicidad}

\section{Productos Herbalife ${ }^{\circledR}$, Omnilife $^{\circledR}$ y Kromasol $^{\circledR}$ (HOK)}

Los productos de estas compañías, que promueven el «bienestar físico y emocional», apoyan en la reducción de peso. Cada compañía reporta ventas de varios millones de dólares, Herbalife ${ }^{\circledR}$ tiene presencia en más de 60 países, Omnilife ${ }^{\circledR}$ en 22 países y $\mathrm{Kromasol}^{\circledR}$ en 18 países $^{14,19-21}$. Muy en particular, Herbalife ${ }^{\circledR}$ es una empresa que producen diferentes productos para pérdida de peso y diversos SD. Los productos fabricados se exportan a más de 50 países de todo el mundo. En 2019, la compañía reportó ventas netas de 1.2 billón de dólares ${ }^{4,22}$. Los SD de las tres marcas son manufacturados especialmente de hierbas seleccionadas, enriquecidas con diferentes nutrientes, oligoelementos, minerales y vitaminas; por lo general el número exacto de productos mezclados, así como su composición exacta, es desconocido. Los pacientes consumen al menos tres productos de cualquiera de las tres marcas líderes, pero llegan a existir hasta 12 productos diferentes de las compañías antes mencionadas que se pueden consumir al mismo tiempo. La mayoría de los pacientes usan productos de HOK principalmente para bajar de peso, los productos pueden incluir en su composición Ephedra sinica y ma huang, este último se ha utilizado durante siglos para inducir la pérdida de peso y se informa que está presente en diversos SD. La efedrina produce una hepatotoxina que se sabe induce lesiones similares a las observadas en los casos estudiados. Según informes ${ }^{14,23}$, en el caso de Herbalife ${ }^{\circledR}$, se encargó de eliminar la efedrina que contenían sus productos después del 2000; los esfuerzos para corroborar esto último con la empresa fueron nulos. Otro compuesto utilizado es la $\mathrm{N}$-nitroso-fenfluramina, contenido en las hierbas chinas chaso u onshido, que también se usa para bajar de peso. Dos hierbas pueden identificarse por el texto en los productos de HOK, uno es el guaraná (Paullinia cupana), la taurina y té verde (Camellia sinensis). El guaraná y la taurina son psicoestimulantes que actúan por medio de la liberación de cafeína con efectos secundarios neurológicos y cardiológicos, la hepatotoxicidad no ha sido reportada. A menudo es difícil convencer al paciente de la toxicidad del producto incriminado. La falta de etiquetado de los 
contenidos de estos productos hace que la evaluación de causalidad sea difícil. Se han reportado casos con datos de hepatotoxicidad después de la ingesta de alguno de los diferentes productos de la marca Herbalife ${ }^{\circledR 3,14,23-26}$, y diversos son los países con datos de reporte: Suiza ${ }^{3,14}$, Israel ${ }^{27}$, España ${ }^{28-30}$, Argentina ${ }^{31}$, Islandia ${ }^{24}$, EE.UU.,32 y Venezuela ${ }^{33}$. Con respecto a los productos de $\mathrm{Kromaso}^{\circledR}$ y $\mathrm{Omnilife}^{\circledR}$, a día de hoy no existen estudios sobre los efectos adversos causados por sus productos, como ha sido en el caso de Herbalife ${ }^{\circledR}$; sin embargo, varios de los productos de $\mathrm{Kromaso}^{\circledR}$, dentro de los diversos compuestos que podemos destacar, tienen potencial para generar efectos a largo plazo. Se encuentran altas cantidades de fructuosa; existen estudios que han demostrado que una alta ingesta de fructosa puede ocasionar efectos perjudiciales, como: sobrepeso, obesidad, resistencia insulínica, diabetes tipo 2, dislipidemias, hiperuricemia, hígado graso no alcohólico y daños renales ${ }^{34-37}$. Por otro lado, a pesar de sus conocidos beneficios, el jugo de noni ha demostrado ser causante de daño hepático grave ${ }^{38,39}$. Por otra parte, ingredientes como la sábila o la sucralosa, Kromaso $^{\circledR}$ los utiliza en otras formulaciones de productos de su marca. Con respecto a la sábila, puede decirse que esta, al igual que el noni, ha sido relacionada con el daño hepático ${ }^{40-45}$. A su vez, la sucralosa ${ }^{46}$ es conocida por ser causante de estrés oxidativo, el cual a la larga desarrolla enfermedades terminales como el asma, la diabetes 0 el cáncer.

\section{Té verde (Camellia sinensis)}

El té verde (Camellia sinensis) 1,4,47 es una bebida muy popular, consumida en todo el mundo durante siglos. Dependiendo del procesamiento, especialmente el "grado de fermentación", el té puede obtener diferencias en sabor, color y composición. El té verde contiene alcaloides de metilxantina (cafeína, teofilina, teobromina), los polifenoles se consideran las principales moléculas bioactivas del té. En las últimas décadas, el consumo del té verde ha aumentado, por sus beneficios para la salud; estudios en humanos no han demostrado un efecto sobre la pérdida de peso, siendo la indicación principal para su consumo. Sin embargo, se ha desarrollado una gran cantidad de productos comerciales que contienen extracto del mismo té que se anuncian como agentes para perder peso. La infusión de té verde es ampliamente consumida, se han reportado casos de daño hepático después de la ingesta de infusión, principalmente los extractos. El primer informe ${ }^{48}$ sobre daño hepático relacionado con la ingesta de extracto de té verde fue en 1999. A partir de entonces se han documentado diversos casos de lesiones hepáticas relacionadas con la ingesta de diferentes extractos de té verde ${ }^{3-5,17,49,50}$. Desde 2006 ha habido más de 50 informes en la literatura médica ${ }^{51,52}$ de lesión hepática aguda con ictericia atribuida a extractos de té verde. Hay casos reportados de hepatotoxicidad debido a extractos de té verde en países como EE.UU., Reino Unido y Australia. En 2008, la Farmacopea de los Estados Unidos ${ }^{53}$ evaluó 34 informes de lesión hepática vinculada a extracto de té verde; 27 fueron etiquetados como probables y los 7 casos restantes inducidos por extractos de té verde. Uno de estos pacientes murió, lo que indica que este tipo de hepatotoxicidad puede tener resultados gra$v \mathrm{~s}^{53}$. Los productos de HOK que contienen dicho extracto, generalmente su etiqueta de advertencia en la monografía de calidad para extracto de té verde, no llega a estar sustentada por varias razones: falta de informes de efectos adversos por prolongado consumo, falta de datos epidemiológicos y la escasez de información sobre la calidad de las preparaciones específicamente vinculada a daño hepático. Los pacientes con daño hepático atribuido a extracto de té verde presentaron una enfermedad característica similar a la hepatitis aguda que ocurre de uno a tres meses de comenzar a consumir el producto. La enfermedad generalmente puede autolimitarse, pero se han reportado casos fatales en hasta un $10 \%$, generalmente aquellos que presentaron lesión hepatocelular aguda e ictericia ${ }^{1}$. En una revisión realizada por Mazzantion de 34 casos publicados ${ }^{51}$, siete tuvieron un cambio positivo debido a la suspensión de los extractos té verde. En la mayoría de los informes de hepatotoxicidad, la dosis humana de extracto de té verde (generalmente menos de $12 \mathrm{mg} / \mathrm{kg}$ al día) no parecía ser excesiva 0 en el rango que podría tener toxicidad directa (estimada para los humanos en $30-90 \mathrm{mg} /$ $\mathrm{kg})^{1,4,54}$.

\section{Ácido linoleico CONJUGado}

El ácido linoleico conjugado (ALC) es un ácido graso poliinsaturado omega-6, es un SD que ha demostrado que causa reducción en la masa de grasa corporal. También se ha demostrado que estimula las respuestas inmunitarias, mejorar la sensibilidad a la insulina y modifica el metabolismo de los lípidos. A pesar de los beneficios atribuidos al ALC, a la fecha se han reportado casos de daño hepático. Todos los pacientes desarrollaron daño hepático de tipo hepatocelular 
después de tomar ALC para ayudar en la pérdida de peso y la reducción de grasa corporal, teniendo resolución completa después de la interrupción de ALC $4,55,56$.

\section{ÁcIDo ÚsNICO}

El ácido úsnico se encuentra únicamente en líquenes, y es especialmente abundante en géneros como Alectoria, Cladonia, Usnea, Lecanora, Ramalina y Evernia. El ácido úsnico funciona como un desacoplador de la cadena respiratoria mitocondrial y se cree que estimula la oxidación del combustible y aumenta las tasas metabólicas, lo que podría influir en la pérdida de peso. Sin embargo, este mecanismo también podría inducir lesiones mitocondriales, en hepatocitos y muerte. Se han reportado varios casos ${ }^{57-59}$ de insuficiencia hepática aguda relacionada sola o con una combinación de SD con ácido úsnico, incluyendo casos que requieren trasplante de hígado. El tiempo de inicio de la lesión hepática generalmente ocurre dentro de los primeros tres meses de consumo. Todos se recuperaron espontáneamente después de suspender el uso del producto; los resultados de las pruebas hepáticas, así como los síntomas, se normalizaron en cuatro meses.

\section{GarcinIA CAMBogIA}

La Garcinia cambogia ${ }^{4,60,61}$ se emplea en suplementos a base de plantas ampliamente promovidos para la pérdida de peso. Ha sido implicada en la hepatotoxicidad en pacientes que toman SD que contiene una variedad de ingredientes y la incluyen.

\section{Diagnóstico de lesión hepática inducida por suplementos dietéticos y herbales}

El diagnóstico de daño hepático inducido por SDH se basa en gran medida en una historia clínica compatible con el consumo de SD, desafortunadamente no existen pruebas de diagnóstico específicas y el patrón de lesión puede ser compatible prácticamente con cualquier enfermedad hepática aguda o crónica. La biopsia hepática puede descartar una lesión hepática crónica y proporcionar evidencia a favor o en contra de otras enfermedades hepáticas ${ }^{1}$.

\section{Resultados}

En la búsqueda de bibliografía sobre el tema en cuestión se pudo observar que la mayoría de los artículos están en idioma ingles y solamente existe uno en español. Es de suma importancia saber que los suplementos son extractos de alimentos deshidratados y naturales, así como de hierbas, vegetales y concentrados de fruta, y pueden o no integrar vitaminas y minerales. Su único objetivo es incrementar la ingesta dietética, completarla o suplir a otros alimentos para obtener sus nutrientes. Si bien la venta de suplementos es abierta a todo el público, algunos foros de salud anticipan que no todas las personas pueden consumirlos; se recomienda supervisión y vigilancia médica previa a su compra. Es importante reconocer estos suplementos alimenticios y considerar sus indicaciones, quiénes pueden consumirlas, cuáles son los efectos adversos que esperar a los beneficios en consumo cotidiano de estas sustancias y tener un indicador medible del grado de beneficio de consumir estos suplementos de manera cotidiana y una revisión periódica con el médico que abarque condiciones físicas, biológicas, psicológicas y sociales para poder determinar el grado de beneficio o en su caso de efectos atribuibles al consumo de estas sustancias.

\section{Discusión}

Los SD no están regulados, como en el caso de los alimentos y tampoco están sujetos a evaluaciones precomercialización y poscomercialización, requisitos de seguridad o eficacia como lo hacen con los medicamentos. Para detectar el factor que contribuye a la hepatotoxicidad, se debe realizar una historia clínica exhaustiva del paciente, solicitando la cronología de la ingesta de medicamentos y SD. En casos reportados por hepatotoxicidad por SD existe un severo subregistro, lo que apunta a una importante problema de salud relacionado con el consumo de SD. Dada la eficacia no probada y el alto número de casos de daño hepático debido a SD, además de la gravedad con riesgo de insuficiencia hepática aguda y posterior muerte o trasplante, los organismos reguladores competentes deben emplear una reglamentación más estricta para la verificación y venta de estos productos. Debe haber un esfuerzo conjunto entre médicos y autoridades sanitarias para identificar datos de hepatotoxicidad, y poder educar a la población sobre los riesgos del consumo de SD y su pronta suspensión. Se necesitan enfoques más rigurosos para evaluar las causas de daño hepático; además, la documentación de datos de casos debe ser completa y sistemática. Los casos deben catalogarse como daño hepático inducido por hierbas o hígado con lesión por SD. 


\section{Conclusión}

Los problemas por la lesión hepática relacionada con SDH plantean varios desafíos de investigación importantes que, cuando se lleguen abordar, ayudarán considerablemente en la comprensión, el control y la prevención de este problema emergente. Los mecanismos por los cuales el SDH causa daño hepático no están claros y requieren una amplia investigación. Identificar la composición química de los SDH es un paso clave para determinar los ingredientes responsables, pero esto debe ser seguido por estudios de las propiedades tóxicas, de los compuestos específicos, utilizando biología celular, cultivos celulares y modelos animales.

\section{Financiamiento}

Los autores no recibieron patrocinio para llevar a cabo este artículo.

\section{Conflicto de intereses}

Los autores declaran no tener conflicto de intereses alguno.

\section{Responsabilidades éticas}

Protección de personas y animales. Los autores declaran que para esta investigación no se han realizado experimentos en seres humanos ni en animales.

Confidencialidad de los datos. Los autores declaran que en este artículo no aparecen datos de pacientes.

Derecho a la privacidad y consentimiento informado. Los autores declaran que en este artículo no aparecen datos de pacientes.

\section{Bibliografía}

1. Navarro V, Khan I, Björnsson E, Seeff L, Serrano J, et al. Liver injury from herbal and dietary supplements. Hepatology. 2017;65(1):363-73.

2. Ernst E, Hung K. Great expectations: what do patients using complementary and alternative medicine hope for? Patient. 2011;4(2):89-101.

3. Stickel F, Shouval D. Hepatotoxicity of herbal and dietary supplements: an update. Arch Toxicol. 2015;89(6):851-65.

4. García M, Robles M, Ortega A, Medina I, Andrade R. Hepatotoxicity by dietary supplements: A tabular listing and clinical characteristics. Int J Mol Sci. 2016;17(4):537.

5. Stickel F, Kessebohm K, Weimann R, Seitz K. Review of liver injury associated with dietary supplements. Liver Int. 2011;31(5):595-605.

6. Navarro V, Barnhart H, Bonkovsky H, Davern T, Fontana R, Grant L, et al. Liver injury from herbals and dietary supplements in the U.S. Drug-Induced Liver Injury Network. Hepatology. 2014;60(4):1399-408.

7. Andrade R, Lucena M, Fernández M, Palaez G, Pachkoria K, García-Ruiz E, et al. Spanish Group for the Study of Drug-Induced liver Disease. Drug-induced liver injury: an analysis of 461 incidences submitted to the Spanish Registry over a 10-year period. Gastroenterology. 2005;129(2):512-21.
8. The Lancet Gastroenterology Hepatology. Herbal assault: liver toxicity of herbal and dietary supplements. Lancet Gastroenterol Hepatol. 2018;3:141.

9. Wai C, Tan B, Chan C, Sutedja D, Lee Y, Khor C, et al. Drug-induced liver injury at an Asian center: a prospective study. Liver Int. 2007(4);27:465-74.

10. Suk K, Kim D, Kim C, Park S, Yoon J, Kim YS, et al. A prospective nationwide study of drug-induced liver injury in Korea. Am J Gastroenterol. 2012;107(9):1380-7.

11. Zhou Y, Yang L, Liao Z, He X, Zhou Y, Guo H, et al. Epidemiology of drug-induced liver injury in China: a systematic analysis of the Chinese literature including 21,789 patients. Eur J Gastroenterol Hepatol. 2013;25(7):825-9.

12. Devarbhavi H, Dierkhising R, Kremers W, Kremers W, Sandeep M, Karanth $\mathrm{D}$, et al. Single-center experience with drug-induced liver injury from India: causes, outcome, prognosis, and predictors of mortality. Am J Gastroenterol. 2010;105(11):2396-404.

13. Björnsson $E$, Bergman $\mathrm{O}$, Björnsson $\mathrm{H}$, Kyaran $\mathrm{R}$, Olafsson $\mathrm{S}$. Incidence, presentation, and outcomes in patients with drug-induced liver injury in the general population of Iceland. Gastroenterology. 2013;144(7):1419-25.

14. Schoepfer A, Engel A, Fattinger K, Marbet U, Criblez D, Reichen J et al. Herbal does not mean innocuous: Ten cases of severe hepatotoxicity associated with dietary supplements from Herbalife products. J Hepatol. 2007;47(4):521-6.

15. Marignani M, Gallina S, Di Fonzo M, Deli I, Begini P, Gigante E, et al. Use and safety perception of herbal remedies in patients with liver/biliary tract disorders: an Italian study. J Clin Gastroenterol. 2010;44(Suppl 1):54-7.

16. Strader D, Bacon B, Lindsay K, La Brecque DR, Morgan T, Wright EC, et al. Use of complementary and alternative medicine in patients with liver disease. Am J Gastroenterol. 2002;97(9):2391-7.

17. García M, Borraz Y, Lucena M, Peláez G, Salmerón J, et al. Hepatotoxicidad secundaria a "productos naturales": análisis de los casos notificados al Registro Español de Hepatotoxicidad. Rev Esp Enferm Dig (Madrid). 2008;100(11):688-95

18. Seef L, Stickel F, Navarro V. Hepatotoxicity of herbals and dietary supplements. Drug induced liver disease. 3 ed. Ámsterdam, Países Bajos: Elsevier; 2013. pp. 631-658.

19. Herbalife Nutrition. Financial Indormation [Internet]. Herbalife Nutrition [consultado: 27/03/2020]. Disponible en: https://ir.herbalife.com/financial-information

20. Conoce Kromasol [Internet]. Kromasol [consultado: 27/03/20. Disponible en: https://kromasol.com/empresa

21. Otega R. Bienvenidos al mundo Omnilife Ya estamos en 22 Países y seguimos Creciendo. [Internet]. RIOENRED [consultado: 27/03/20]. Disponible en: http://rioenred.com/profiles/blogs/bienvenidos-al-mundo-omnilife

22. Herbalife Nutrition Reports Record-Breaking Quarterly Volume Point Results and Provides Initial Full Year 2020 Guidance [Internet]. Herbalife Nutrition [consultado: 27/03/20]. Disponible en: https://ir.herbalife.com/ static-files/e7852f47-7927-42fb-9666-95e691b99d21

23. Bonkovsky $\mathrm{H}$. Hepatotoxicity associated with supplements containing Chinese green tea (Camellia sinensis). Ann Intern Med. 2006;144(5):68-71.

24. Jóhannsson M, Ormarsdóttir S, Olafsson S. Hepatotoxicity associated with the use of Herbalife. Laeknabladid. 2010;96(3):167-72.

25. Teschke R, Zhang L, Melzer L, Schulze J, Eickhoff A. Green tea extract and the risk of drug-induced liver injury. Exp Opin Drug Metab Toxicol. 2014;10(12):1663-76.

26. Dante J, Aguilar D, Almanza C. Hepatotoxicidad, suplementos naturistas. Presentación de dos casos clínicos. Rev Sanid Milit Mex. 2012;66(2):100-4.

27. Elinav E, Pinsker G, Safadi R, Pappo O, Bromberg M, Anis E, et al. Association between consumption of Herbalife nutritional supplements and acute hepatotoxicity. J Hepatol. 2007;47(4):514-20.

28. Duque J, Ferreiro J, Salgueiro E, Manso G. Hepatotoxicity associated with the consumption of herbal slimming products. Med Clin (Barc). 2007;128:238-9.

29. Manso G, López L, Duque J, Salgueiro E. Spanish reports of hepatotoxicity associated with Herbalife products. J Hepatol. 2008;49(2):289-90.

30. Manso G, López L, Salgueiro M, Duque J, Jimeno F, Andrade RJ, et al. Continuous reporting of new cases in Spain supports the relationship between Herbalife ${ }^{\circledR}$ products and liver injury. Pharmacoepidemiol. Drug Saf. 2011;20(10):1080-7

31. Chao S, Anders M, Turbay M, Olaiz E, Mc Cormack L, Mastai R. Hepatitis aguda asociada al consumo de Herbalife ${ }^{\circledR}$ a propósito de un caso. Acta Gastroenterol Latinoam. 2008;38(4):274-7.

32. Chen G, Ramanathan V, Law D, Funchain P, Chen G, French S, et al. Acute liver injury induced by weight-loss herbal supplements. World $\mathrm{J}$ Hepatol. 2010;2(11):410-5.

33. Mengual E, Lizarzábal M, Ruiz M, Silva N, Andrade R, Lucena M, et al. Reporte de casos de lesión hepática inducida por medicamentos en un centro hospitalario de referencia del estado Zulia, Venezuela. Investig Clin. 2015;56(1):3-12.

34. Tappya L, Lêc K. Does fructose consumption contribute to non-alcoholic fatty liver disease? Clin Res Hepatol Gastroenterol. 2012;36(6):554-60. 
35. Zhang $Y$, An $T$, Cheng $R$, Zhou Q, Huang $Y$, Zhang J, et al. Very high fructose intake increases serum LDL-cholesterol and total cholesterol: A meta-analysis of controlled feeding trials. J Nutr. 2013;143(9): 1391-8.

36. Johnson R, Sanchez L, Nakagawa $T$. The effect of fructose on renal biology and disease. J Am Soc Nephrol. 2010;21(12):2036-9.

37. Bantle J. Dietary fructose and metabolic syndrome and diabetes. J Nutr. 2009;139(6):1263-8.

38. López-Cepero J, Lerma S, Fernández M, Amaya A. Hepatotoxicidad grave asociada al consumo de Noni (Morinda citrifolia). Rev Esp Enferm Dig (Madrid). 2007;99(3):173-81.

39. Garrido F, Muñoz R, Muñoz C, Delgado P, Fernández I, Castellano G Acute liver failure in a patient consuming Herbalife products and Noni juice. Rev Esp Enferm Dig (Madrid). 2015;107(4):247-8.

40. Moreira V, Garrido E. Hepatotoxicidad por productos de herboristería. Rev Esp Enferm Dig (Madrid). 2013;105(7):433.

41. Rabe C, Musch A, Schirmacher P, Kruis W, Hoffmann R. Acute hepatitis induced by an aloe vera preparation: A case report. World J Gastroenterol. 2005;11(2):303-4.

42. Yang H, Kim D, Kim Y, Kim B, Sohn K, Choi MJ, et al. Aloe induced toxic hepatitis. J Korean Med Sci. 2010;25(3):492-5.

43. Kanat O, Ozet A, Ataergin S. Aloe vera-induced acute toxic hepatitis in a healthy young man. Eur J Intern Med. 2006;17(8):589.

44. Bottenberg M, Wall G, Harvey R, Habib S. Oral aloe vera-induced hepatitis. Ann Pharmacother. 2007:41(10):1740-3.

45. Curciarello J, De Ortúzar S, Borzi S, Bosia D. Hepatitis aguda grave asociada al consumo de aloe vera. Gastroenterol Hepatol. 2008;31(7) 436-8.

46. Hua Y, Sreejayan N. Sucralose and its effect on levels of nitric oxide synthase in endothelial cells [Internet]. University of Wyoming; 2014 [consultado: 23/03/20]. Disponible en: https://repository.uwyo.edu/ ugrd/2014 UGRD/Schedule/59

47. Wolfram S, Wang Y, Thielecke F. Anti-obesity effects of green tea: from bedside to bench. Mol Nutr Food Res. 2006;50(2):176-87.

48. Gavilan J, Bermudez F, Salgado F, Pena D. Phytotherapy and hepatitis. Rev Clin Esp. 1999;199(10):693-4.
49. Abu el Wafa Y, Benavente A, Talavera A, Pérez M, Ramos J. Acute hepatitis induced by Camellia sinensis (green tea). An Med Interna. 2005;22(6):298.

50. Dueñas C, Fabregas S, Durández R. Hepatotoxicity due to Camelia sinensis. Med Clin. 2004;122(17):677-8.

51. Mazzanti G, Menniti-Ippolito F, Moro P, Cassetti F, Raschetti R, Santuccio $\mathrm{C}$, et al. Hepatotoxicity from green tea: a review of the literature and two unpublished cases. Eur J Clin Pharmacol. 2009;65(4):331-41.

52. Mazzanti G, Di Sotto A, Vitalone A. Hepatotoxicity of green tea: an update. Arch Toxicol. 2015;89(8):1175-91.

53. Sarma D, Barrett M, Chavez M, Gardiner P, Ko R, Mahady GB, et al. Safety of green tea extracts: a systematic review by the US Pharmacopeia. Drug Saf. 2008;31(6):469-84.

54. García S, Sáez F, Gento E, López A, Arias L. Acute hepatitis associated with Camellia thea and Orthosiphon stamineus ingestión. Gastroenterol Hepatol. 2004;27(9):559-60.

55. Favreau J, Ryu M, Braunstein G, Orshansky G, Park S, Coody GL, et al. Severe hepatotoxicity associated with the dietary supplement LipoKinetix. Ann Intern Med. 2002;136(8):590-5.

56. Durazo F, Lassman C, Han S, Saab S, Lee N, Kawano M, et al. Fulminant liver failure due to usnic acid for weight loss. Am J Gastroenterol. 2004;99(5):950-2

57. Sanchez W, Maple J, Burgart L, Kamath P. Severe hepatotoxicity associated with use of a dietary supplement containing usnic acid. Mayo Clin Proc. 2006;81(4):541-4.

58. Estes J, Stolpman D, Olyaei A, Corless C, Ham J, Schwartz JM, et al. High prevalence of potentially hepatotoxic herbal supplement use in patients with fulminant hepatic failure. Arch Surg. 2003;138(8):852-8.

59. Neff G, Reddy K, Durazo F, Meyer D, Marrero R, Kaplowitz N, et al. Severe hepatotoxicity associated with the use of weight loss diet supplements containing ma huang or usnic acid. J Hepatol. 2004;41(6):1062-4.

60. Corey R, Werner K, Singer A, Moss A, Smith M, Noelting J, et al. Acute liver failure associated with Garcinia cambogia use. Ann Hepatol. 2015;15(1):123-6.

61. Melendez J, Snipelisky D, Matcha G, Stancampiano F. Acute hepatitis induced by pure Garcinia cambogia. J Clin Gastroenterol. 2015;49(5):449-50. 